\title{
ACTITUDES Y CONOCIMIENTOS ANTE LA OCURRENCIA DE SISMOS EN PERSONAL DE SALUD EN ICA, 2016
}

\author{
Attitudes And Knowledge To The Occurrence Of \\ Earthquakes In Health Personnel Of Ica, 2016
}

César Miguel Quispe-Nolazco, ${ }^{1, a}$, Mary Chris Quispe-Bravo ${ }^{1, a}$, Ángel Anicama Hernández $^{2, a, b}$ 1.Dirección Regional de Salud Ayacucho. Ayacucho, Perú 2. Hospital Regional de Ica. Ica, Perú a. Médico Cirujano b.Médico Neurologo.

Correspondencia:

César Miguel Quispe-Nolazco Dirección: Urb. Guzmán Barrios A10 Chincha-Ica.

Correo electrónico:

cquispe.n4@gmail.com

Teléfono: 956018000

Conflicto De Intereses: No declarados.

\section{Financiamiento: Autofinanciado.}

Recibido: 13-02-2017

Aceptado: 19-02-2017

Publicado: 06-03-2017

\section{CITAR COMO:}

Quispe-Nolazco CM, QuispeBravo MC, Anicama-Hernández A. Actitudes Y Conocimientos Ante La Ocurrencia De Sismos En Personal De Salud En Ica, 2016. Rev méd panacea. 2017; 6(1): 17-22.

\begin{abstract}
RESUMEN
Introducción: El ser humano está expuesto a muchas amenazas según el entorno que lo rodea; el Perú no es la excepción; las experiencias ante las consecuencias de los desastres originados por la naturaleza que vienen ocurriendo desde los últimos años, han puesto en relieve la vulnerabilidad de la salud pública, donde las instituciones incumplen con las acciones de prevención y la poca preparación de la población para hacer frente a estos desastres naturales. En ello como respuesta, el sector salud forma parte de la toma de decisiones dentro del comité operativo de emergencia para la coordinación intersectorial en situaciones de crisis, donde se altera el equilibrio de la naturaleza y fraccionan al grupo humano residente en distintos escenarios. Objetivos: Determinar la relación entre las actitudes y conocimientos ante la ocurrencia de sismos en estudiantes de medicina, y médicos del Hospital Regional de Ica. Material y métodos: Nivel de investigación relacional, tipo descriptivo, prospectivo y transversal. Realizado mediante una encuesta auto aplicada. Análisis mediante SPSS 24.0 aplicando regresión lineal y chi-cuadrado $(p<0.05)$ y análisis multivariado mediante el modelo de regresión logística. Resultados: Como factores de riesgo se tiene el grado académico, la convivencia con personas con discapacidad, la falta de experiencia en un sismo, no pertenecer a una brigada, no haber llevado un curso en prevención de desastres y el bajo conocimiento en el tema.
\end{abstract}

Palabras clave: Sismos, medidas preventivas, desastres, Médicos, Estudiantes Premédicos .

\begin{abstract}
Introduction: The human being is exposed to many threats according to the surrounding environment and Peru is no exception. The experiences of the natural disasters that have been occurring since the last years have highlighted the vulnerability of public health, where institutions fail to comply with prevention actions and the lack of preparedness of the population to cope to these natural disasters. As a response, the health sector is part of the decision-making process within the emergency operational committee for intersectoral coordination in crisis situations, where the balance of nature is altered and the human group residing in different scenarios is divided. Objetive: To determine the relationship between attitudes and knowledge regarding the occurrence of earthquakes in medical students and physicians at the Regional Hospital of Ica. Materials and Methods: Relational research level, analytical type, prospective and transversal. Made through a self-administered survey. Analysis using SPSS 24.0 applying linear and chi-square regression $(p<0.05)$ and multivariate analysis using the logistic regression model. Results: Risk factors include academic degree, coexistence with people with disabilities, lack of experience in an earthquake, not joing to a brigade, not having taken a course in disaster prevention and low knowledge in the subject.
\end{abstract}

Keywords : Seisms, prevention \& control, disasters, Physicians, Students, Premedical. 


\section{INTRODUCCIÓN}

Diariamente el ser humano está expuesto a muchas amenazas según el entorno que lo rodea. Perú no es la excepción; las experiencias ante las consecuencias de los desastres originados por la naturaleza que vienen ocurriendo desde los últimos años en nuestro país, han puesto en relieve la vulnerabilidad de la salud pública(1).

Uno de los últimos sismos originados en el país fue el terremoto del 15 de Agosto del 2007 con grandes pérdidas en muchos ámbitos, es comparado con el sismo de Chile ocurrido el primero de abril del 2014, que al tener las mismas características o mayores, las secuelas por el sismo fueron mucho menores, entonces cuáles son las causas, según autoridades chilenas, destacan el comportamiento ejemplar de la población, así como su cultura cívica que les permitió realizar una evacuación tranquila y masiva (2).

En Perú, las instituciones incumplen con las acciones de prevención dispuestos por ley, lo que da como resultado el no contar con un plan de contingencia, evacuación y la determinación de zonas de seguridad actualizadas y acordes a la realidad donde se encuentren, a ello agregamos la poca preparación de la población para hacer frente a estos desastres naturales, la falta de cooperación en los simulacros y la escasa organización e información en la comunidad que dará como resultado una vulnerabilidad. El sector salud tiene un papel participativo en ello, donde forma parte de la toma de decisiones dentro del comité operativo de emergencia, siendo este la instancia de coordinación intersectorial en situaciones de crisis (3).

En estudios realizados, Ostad Taghizadeh, y cols. (4), encuentran conocimientos correctos y actitudes positivas pero que en la práctica no se refleja, True y cols. (5), Jasper y cols. (6) y Sergio Endo y cols. (7), encuentran que alrededor del $50 \%$ desconocen sobre el tema, y que al menos un 20\% tendría algún dominio en situaciones de desastres.

Entre las variables de protección asociadas ante la respuesta adecuada ante sismo, True y cols. (5) y Tekeli-Yeșil y cols. (8), hallan la edad mayor de 44 años, del mismo modo, los padres solteros y presencia de niños en el hogar, Jasper y cols. (6), demuestran solo el $47 \%$ de los internos de medicina recibe entrenamiento formal en la preparación ante desastres, por el contrario Abhinav Sinha y cols. (9), les queda en claro que ningún conocimiento fue impartido formalmente a los estudiantes con respecto a la preparación y mitigación de desastres de emergencia, sino que sus conocimientos se debe a un aprendizaje auto-adquirido, asimismo, Ocharán Portugal (10), encuentra en los que forman parte de una brigada de emergencia, una asociación significativa para actitudes conocimientos adecuados.

Puesto que nunca se está suficientemente preparado para cuando ocurra una situación de emergencia, donde se altera el equilibrio de la naturaleza y fraccionan al grupo humano residente en distintos escenarios, se tiene la interrogante de saber si se cuenta con el personal de salud presto a reaccionar de manera eficaz y eficiente a las contingencias de un evento natural, siendo el objetivo de esta investigación, determinar las actitudes y conocimientos éntrelos estudiantes de medicina y médicos así como conocer los factores de riesgo relacionados a una actitud inadecuada, con la finalidad de poder establecer una cultura de la prevención.

\section{MATERIALES Y MÉTODOS}

Estudio de nivel relacional, tipo prospectivo, transversal y descriptivo. El universo está conformado por la población de los estudiantes de la facultad de Medicina Humana de la Universidad San Luis Gonzaga y los médicos del Hospital Regional de Ica, un total $(\mathrm{N}=839)$. Se escogerán a los participantes de manera aleatoria sistemática, ya que se estará contando con una lista de toda la población, usando una muestra de una proporción estratificada. La muestra de médicos del Hospital Regional de Ica es de 111 personas y estudiantes de Medicina FMH-DAC de 341 personas.

Entre los criterios de inclusión se tendrán como parte del estudio a los alumnos matriculados regularmente en la Facultad de Medicina
Humana Daniel A. Carrión del año académico 2015-II, médicos nombrados y contratados, y Médicos residentes que trabajen actualmente en el Hospital Regional de Ica al año 2015 y los que acepten responder el cuestionario y firmar el consentimiento informado.

Entre los criterios de exclusión se tiene a los alumnos suspendidos por exceso de matrículas y al personal de no médico, seguridad, limpieza ni administrativo del Hospital Regional de Ica.

Se realiza la recolección de datos, explicando a los alumnos/médicos sobre la investigación, firma del consentimiento informado y compromiso de participación, ya sea la ficha en físico o de manera virtual (Tecnología de Google Forms), seguidamente se aplica el cuestionario con una duración promedio de 30 minutos, una vez terminado se procede a la calificación de las pruebas calculando los indicadores correspondientes y exponiendo los resultados en una base de datos para su posterior análisis utilizando un software estadístico, SPSS v.24.0. La ficha de recolección de datos sociodemográficos, un cuestionario Likert, un examen de conocimiento y una hoja de consentimiento informado, instrumentos validados mediante una medición de confiabilidad calculado mediante el Alfa de Crombach y el método de Dos Mitades (Coeficiente > 0.7), además de realizar la validación por la opinión de expertos por la especialidad, evaluando cada reactivo del instrumento.

Para el análisis univariado, se realiza análisis de tendencia central, en el análisis bivariado se aplica el Análisis correlacional para detectar grado de asociación, análisis de regresión lineal para detectar capacidad predictiva, análisis multivariado mediante el modelo de regresión logística para determinar los factores de riesgo que implican con respecto a la variable dependiente.

Se tiene en el estudio como principio ético, la confidencialidad, donde la información obtenida se manejó de forma discreta y confidencial con uso exclusivo de la investigación, no se divulga en los resultados los nombres de los participantes asignándoles un código de identificación y la beneficencia, en primera instancia a la institución, la cual podrá conocer la realidad de la situación sobre prevención en sismos, los siguientes beneficiarios serán los alumnos y personal médico, ya que una vez que las autoridades de la institución obtengan los resultados, estas podrán desarrollar las medidas convenientes que garanticen un mejor desempeño ante ello. Por último, nosotros como investigadores, encontramos en este proyecto la oportunidad de aportar información valiosa a nuestra comunidad, además de la adquisición de experiencia y reconocimiento personal.

\section{RESULTADOS}

La población de estudio fue constituido por 460 personas, entre estudiantes de medicina representado por el $75 \%$ y médicos por el $25 \%$, de la población en estudio, el $54 \%$ tiene actitudes negativas, siendo los estudiantes quienes presentan mayor actitudes negativas ante la ocurrencia de sismos frente a los médicos, un $82 \%$ frente a un $18 \%$ respectivamente. En los conocimientos sobre sismos, el $12 \%$ de los participantes tiene conocimientos inadecuados, siendo los estudiantes quienes presentan mayores conocimientos inadecuados frente a los médicos, un $86 \%$ frente a $14 \%$ respectivamente como se menciona en la Tabla 1.

Al relacionar las variables de riesgo con actitudes negativas en prevención de sismo, se encuentra una asociación significativa con el grado universitario, menores de 26 años, solteros, en los que tienen algún familiar con discapacidad, residencia menor de 20 años en Ica, la falta de experiencia de un terremoto, la no participación en simulacros, los que no pertenecen a una brigada de emergencia o de su institución y en aquellos que no han llevado algún curso de prevención de desastres como se mencionan en las Tabla 2 y Tabla 3. Dentro de los factores intervinientes en relación a los conocimientos en prevención en sismos, encontramos como factores de riesgo, al grado universitario, los que no pertenecen una brigada de emergencia o de su institución, el no haber llevado algún curso en prevención de 
Idesastres como se describe en la Tabla 4 y Tabla 5.

Al tener los resultados por la escala Likert por Actitudes y el test en conocimientos, se obtiene que existe una asociación entre las variables, (p-valor: 0,00 OR: 6,217 IC: 2,871 - 13,460), siendo los conocimientos incorrectos un factor de riesgo tanto para los médicos y universitarios, mostrándose entre 5 y 6 veces más sobre los que tienen conocimientos correctos para presentar una actitud negativa ante la prevención de sismo respectivamente (Médicos: p-valor: 0,030, OR: 5,289 IC: 1,017-27,516) (Universitarios: p-valor: 0,000, OR: 6,016 IC: $2,485-14,564)$.

Dentro de las dimensiones en actitudes y conocimientos frente a la prevención de sismos, presentan de forma adecuada un $86 \%$ en el aspecto general de sismo, $89 \%$ en tipología del comportamiento humano donde incluyendo ideologías y seguridad del participante, $66 \%$ en el punto de medios de comunicación, $79 \%$ en medidas preventivas ante la ocurrencia de sismo; puntos como mochila de emergencia, participación en simulacros, capacitaciones y soporte vital; $83 \%$ en reconocimiento de la responsabilidad Institucional; tanto de la institución de estudio/trabajo como en las brigadas de emergencia - COE; $13 \%$ en plan de contingencia y en $58 \%$ sobre identidad institucional.

El análisis multivariado mediante el modelo de regresión logística, arroja que los factores de riesgo que están presentes para realizar actitudes negativas, se encuentra el grado universitario, , las personas que conviven con personas con discapacidad, la no experiencia vivida de un terremoto, el no pertenecer a una brigada de emergencia, no

Tabla 1: Características Generales de la Población

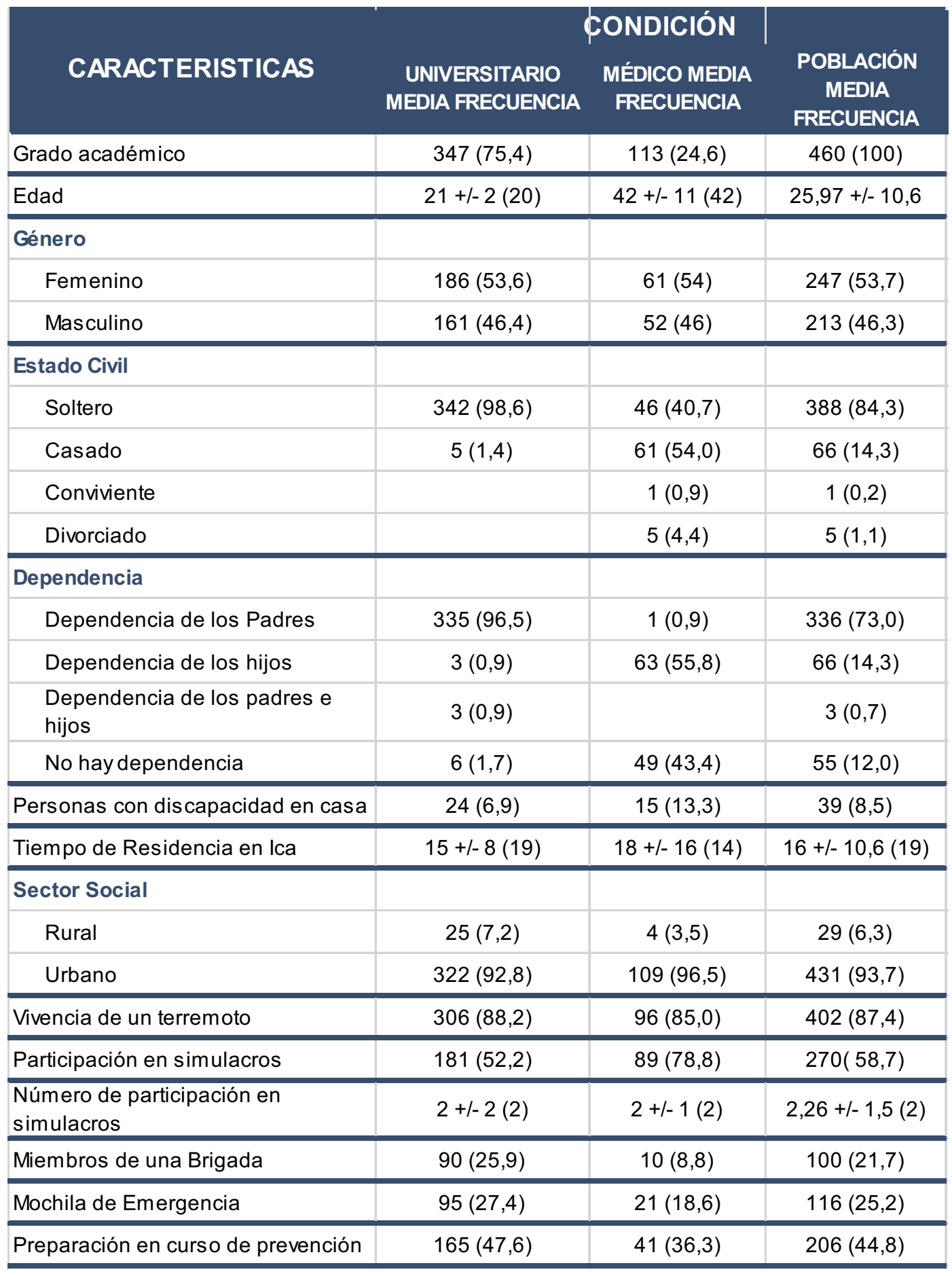


Tabla 2: Grado de Actitud ante la ocurrencia de sismo

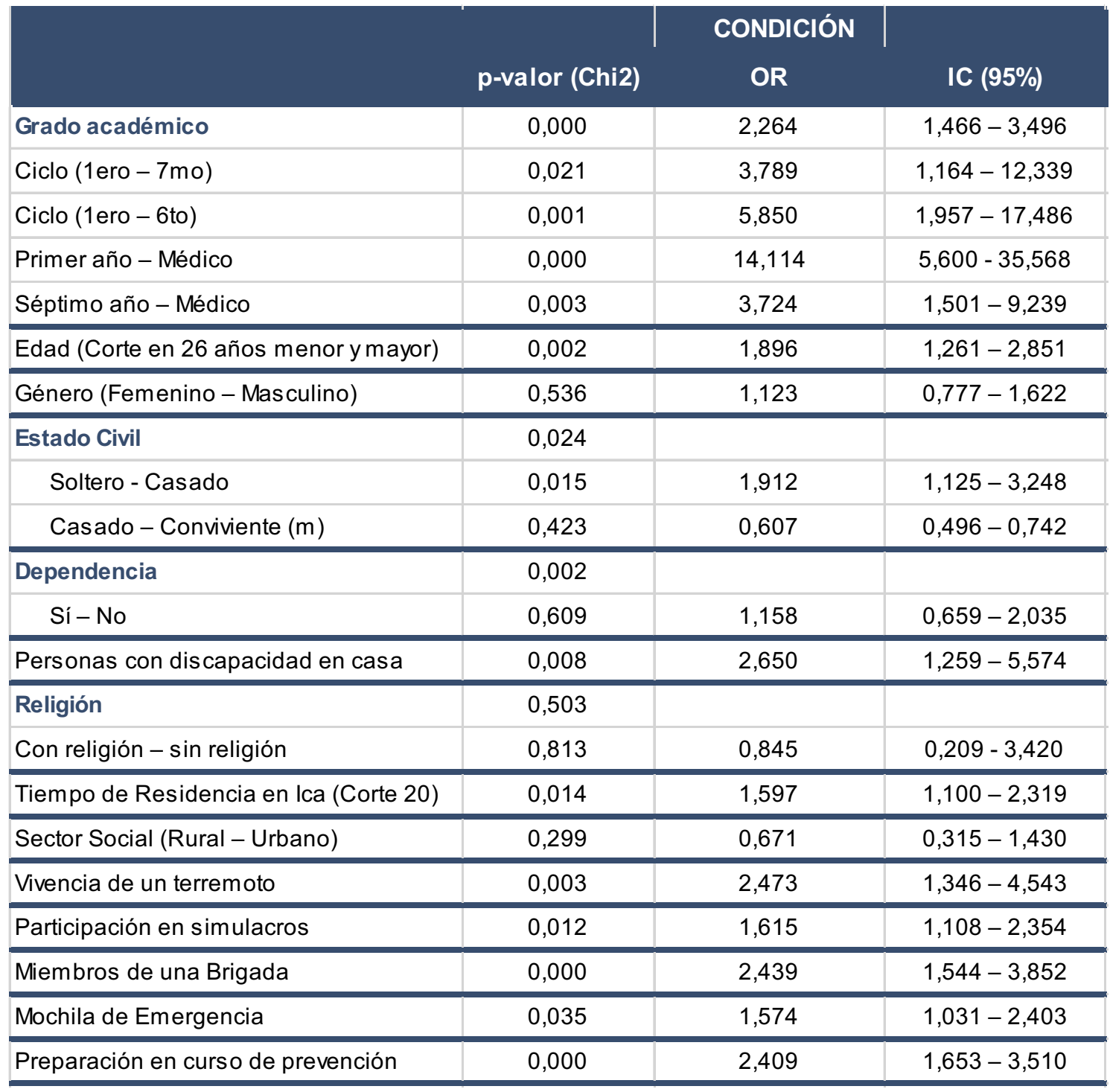

Tabla 3: Grado de actitud ante la ocurrencia de sismo según grado académico

\begin{tabular}{|l|l|c|c|c|}
\hline & \multicolumn{2}{|c|}{ p-valor (Chi2) } & OR & \multicolumn{1}{c|}{ IC (95\%) } \\
\hline \multirow{3}{*}{ ALUMNOS* } & *Tiempo de Residencia en Ica (Corte 20) & 0,140 & 1,385 & $0,898-2,137$ \\
\cline { 2 - 5 } & Vivencia de un terremoto & 0,001 & 3,835 & $1,648-8,920$ \\
\cline { 2 - 5 } & Participación en simulacros & 0,017 & 1,693 & $1,098-2,612$ \\
\cline { 2 - 5 } & Miembros de una Brigada & 0,000 & 3,733 & $2,255-6,180$ \\
\hline & Preparación en curso de prevención & 0,000 & 2,709 & $1,743-4,211$ \\
\hline \multirow{2}{*}{ MÉDICOS** } & **Tiempo Residencia en Ica (Corte 20) & 0,018 & 2,601 & $1,167-5,799$ \\
\cline { 2 - 5 } & Vivencia de un terremoto & 0,456 & 1,481 & $0,525-4,184$ \\
\cline { 2 - 5 } & Participación en simulacros & 0,269 & 0,579 & $0,218-1,536$ \\
\hline & Miembros de una Brigada & 0,452 & 0,609 & $0,166-2,240$ \\
\hline & Preparación en curso de prevención & 0,027 & 2,774 & $1,186-6,488$ \\
\hline
\end{tabular}


tener preparación en algún curso en prevención de desastre por sismo, y tener conocimientos incorrectos de sismo, como se muestra en la Tabla 6.

\section{DISCUSIÓN}

En el trabajo se encuentra que el $45,87 \%$ presenta actitudes positivas ante la prevención de sismos y un $87,61 \%$ tiene buenos conocimientos, siendo los universitarios quienes muestran mayores actitudes negativas y conocimientos incorrectos. En estudios realizados, Ostad Taghizadeh, y cols. (4), encuentran mayores frecuencias con un $90 \%$ en conocimientos correctos y un $97 \%$ de actitudes positivas pero que en la práctica presentan un $41 \%$ de forma correcta, menor fue la frecuencia que encontró, True y cols. (5), donde solo el $53 \%$ encontró con mayor del $75 \%$ de conocimientos correctos; Jasper y cols. (6), observa una insuficiencia de conocimientos en el tema, donde solo un 20\% tendría algún dominio en situaciones de desastres, Ocharán Portugal (10), encuentra el nivel de conocimientos sobre el plan Hospitalario en "Medio" con el 61,8\% y “Bajo" en el 38,2\% de los trabajadores, así como el $40 \%$ de los participantes no están capacitados ante una situación de desastre en el hospital.

De la población de estudio, el $45 \%$ ha llevado un curso, los médicos en un $36 \%$ y en mayor frecuencia con $48 \%$ de los estudiantes, al realizar el análisis multivariado se encuentra que aquellos que no están preparado por cursos en prevención de sismo se muestra como un factor de riesgo para padecer actitudes negativas en prevención de sismos (p-valor: 0,000, OR 2,696 IC: 1,7-4,2), resultados como Jasper y cols. (6),

Tabla 4: Conocimientos en sismos

\begin{tabular}{|l|c|c|c|}
\hline & p-valor (Chi2) & OR & IC (95\%) \\
\hline Grado académico & 0,048 & 2,158 & $0,990-4,707$ \\
\hline Primer año - Médico & 0,000 & 6,563 & $2,676-16,095$ \\
\hline Séptimo año - Médico & 0,066 & 2,983 & $0,891-9,985$ \\
\hline Miembros de una Brigada & 0,028 & 2,586 & $1,076-6,214$ \\
\hline Preparación en curso de prevención & 0,675 & 0,888 & $0,509-1,548$ \\
\hline
\end{tabular}

Tabla 5: Conocimientos en sismos según grado académico

\begin{tabular}{|c|l|c|c|c|}
\hline \multirow{2}{*}{ Alumnos } & & P-valor (Chi2) & OR & IC (95\%) \\
\cline { 2 - 5 } & Miembros de una Brigada & 0,018 & 2,813 & $1,154-6,855$ \\
\hline \multirow{2}{*}{ Médicos } & Preparación en curso de prevención & 0,405 & 0,773 & $0,422-1,417$ \\
\hline & Miembros de una Brigada & 0,361 & 0,922 & $0,872-0,975$ \\
\cline { 2 - 5 } & Preparación en curso de prevención & 0,147 & 4,308 & $0,511-36,321$ \\
\hline
\end{tabular}

Tabla 6: Análisis multivariado mediante el modelo de regresión logística.

\begin{tabular}{|l|l|c|c|c|}
\hline & \multicolumn{2}{c}{ p-valor } & OR & IC (95\%) \\
\hline & Grado académico & 0,009 & 5,868 & $1,559-22,094$ \\
\hline Edad (Corte en 26 años) & 0,648 & 0,783 & $0,274-2,240$ \\
\hline Estado Civil (Soltero - Casado) & 0,644 & 0,796 & $0,301-2,101$ \\
\hline & Dependencia (Sí - No) & 0,127 & 0,459 & $0,169-1,247$ \\
\hline \multirow{2}{*}{ ACTITUDES } & Personas con discapacidad (Sí - No) & 0,006 & 3,204 & $1,403-7,319$ \\
\hline NEGATIVAS & Tiempo de Residencia en Ica (<- >20) & 0,050 & 1,544 & $0,999-2,385$ \\
\hline & Vivencia de un terremoto (No - Sí) & 0,004 & 2,693 & $1,374-5,276$ \\
\hline & Participación en simulacros (No - Sí) & 0,066 & 1,512 & $0,973-2,348$ \\
\hline & Miembros de una Brigada (No - Sí) & 0,006 & 2,136 & $1,241-3,677$ \\
\hline & Preparación en curso de prevención & 0,000 & 2,696 & $1,718-4,229$ \\
\hline & Conocimiento ante prevención de sismo & 0,000 & 4,785 & $2,110-10,853$ \\
\hline
\end{tabular}


demuestran que el $47 \%$ de los internos de medicina recibe entrenamiento formal en la preparación ante desastres en la escuela de medicina; $64 \%$ de estos programas de capacitación incluyen algún tipo de simulación y que el $60 \%$ de los internos no tiene ningún tipo de preparación, Corrigan y Samrasinghe (11), quienes establecieron los conocimientos en desastres en el personal de salud de un hospital, encuentran que el 59\% habían recibido previamente educación de desastres; $38 \%$ asistieron a un ejercicio de simulación de desastres, y $13 \%$ habían respondido a un desastre real, donde tienen mejor respuesta en las simulaciones aquellos que han tenido alguna capacitación previa, por el contrario Abhinav Sinha y cols. (9), al evaluar a los estudiantes de medicina queda en claro que ningún conocimiento fue impartido formalmente a los estudiantes con respecto a la preparación y mitigación de desastres de emergencia, sino que sus conocimientos se debe a experiencias anteriores autoaprendidas y del aprendizaje auto-adquirido sobre desastres; Soffer y. col. (12), encuentran que el hablar con los amigos cercanos y miembros de la familia o lecturas sobre los terremotos, son factores de protección, el cual les instruyen para tomar correcciones las acciones en caso de un terremoto.

Con respecto a la integración en una brigada de emergencia para eventos de sismos, Ocharán Portugal (10), encuentra que el 31\% forma parte de una brigada de emergencia, hallando una asociación significativa, donde el no pertenecer a una brigada $(p=0,036)$ presenta un nivel de conocimiento "Bajo". En nuestro estudio, a través del análisis multivariado, se encuentra como factor de riesgo el no pertenecer a una brigada de emergencia para tener actitudes negativas ante la ocurrencia de sismo ( $p$-valor: 0,006 OR: 2,136 IC:1,2 $3,7)$.

Concluyendo que los factores de riesgo para tener actitudes negativas, es el grado universitario, tener algún familiar con discapacidad, la falta de experiencia previa de un sismo, y el bajo conocimiento de sismo.

Así mismo se encontró como que el no pertenecer a una brigada de emergencia; el C.O.E. (Comité operativo de emergencias) de cada institución; y no llevar algún curso de preparación en desastres, representa un factor de riesgo para tener actitudes negativas, puesto que la educación previa, la participación de ejercicios de simulación y el aprendizaje auto-adquirido se obtiene una mejor preparación.

Demostrando que hay una necesidad de mejorar el nivel de preparación ante desastres, en la formación de las escuelas de medicina desarrollando aspectos que promuevan la retención de conocimientos para estar preparados ante desastres. Fomentar la capacitación continua del personal médico del Hospital Regional con cursos actualizados para optimizar la respuesta ante el aumento de demanda ante la ocurrencia de sismo, así como promover la participación activa en simulacros de manera responsable y seria para obtener una real evaluación de la actitud de estudiantes de la facultad de medicina del personal médico del Hospital Regional. Se tiene como un factor importante la integración a brigadas de emergencia desde primeros años en la Facultad de Medicina para aumentar y afianzar los conocimientos y a la vez mejorar la respuesta ante la ocurrencia de un sismo.

Se tiene limitación en este estudio, la población estudiantil y médica de una sola sede.

\section{BIBLIOGRAFÍA}

1. Organización Panamericana de la Salud. Terremoto de Pisco - Perú. A dos años del sismo, crónica y lecciones aprendidas en el sector salud Washington, D.C: PAHO; 2010.

2. ONEMI - Oficina Nacional de Emergencia del Ministerio del Interior y Seguridad Pública. ONEMI - CHILE; 2014.

3. Salud OPdl. Modelo de organización del sector salud en situaciones de desastre. Biblioteca virtual de desarrollo sostenible y salud ambiental. 2008.

4. Ostad Taghizadeh A, Hosseini M, Navidi I, Mahaki AA, Ammari H, Ardalan A. Knowledge, Attitude and Practice of Tehran's Inhabitants for an Earthquake and Related Determinants. PLOS Currents Disasters. 2012 August. Edition 1;1.

5. True NA, Adedoyin JD, Shofer FS, Hasty EK, Brice JH. Level of Disaster Preparedness in Patients Visiting the Emergency Department: Results of the Civilian Assessment of Readiness for Disaster (CARD) Survey. Prehospital and Disaster Medicine. 2013 January; 28(2): p. 127-131.

6. Jasper E, Berg K, Reid M, Gomella P, Weber D, Schaeffer A, et al. Disaster Preparedness: What Training Do Our Interns Receive During Medical School? American Journal of Medical Quality. 2013 Sep-Oct; 28(5): p. 407-13.

7. Endo S, Fuentes I, Vásquez T, Zavala J. Características del personal del Hospital de Emergencias José Casimiro Ulloa y nivel de conocimiento de medidas de acción durante sismo. Escuela Académico Profesional de Medicina Humana. Facultad de MedicinaUNMSM. 2007.

8. Tekeli-Yeşil S, Dedeoğlu N, Braun-Fahrlaender C, Tanner M. Earthquake awareness and perception of risk among the residents of Istanbul. Natural Hazards. 2011 October; 59(1): p. 427-446.

9. Abhinav S, D.K.P, P.K. K, R. T, A. S. Knowledge, attitude and practice of disaster preparedness and mitigation among medical students. Disaster Prevention and Management. 2008;17(4):p.503-507.

10. Ocharán Portugal JD. Nivel de conocimiento del plan hospitalario y medidas de acción frente a situaciones de desastres en el Hospital Nacional Cayetano Heredia : año 2013. [Trabajo de investigación para optar el Título de Especialista en Medicina de Emergencias y Desastres]. Universidad Mayor de San Marcos Facultad de Medicina Humana Escuela de post-grado. 2013.

11. Corrigan E, Samrasinghe I. Disaster Preparedness in an Australian Urban Trauma Center: Staff Knowledge and Perceptions. Prehospital and Disaster Medicine. 2012 October; 27(5): p. 432-438.

12. Soffer $Y$, Goldberg A, Adini B, Cohen R, Ben-Ezra M, Palgi $Y$, et al. The relationship between demographic/educational parameters and perceptions, knowledge and earthquake mitigation in Israel. Disasters. 2011 January; 35(1): p. 36-44. 